\title{
Sieroepidemiologia delle infezioni da Toxoplasma gondii, virus della Rosolia e Citomegalovirus in una coorte di gravide
}

\author{
Marco Moretti', Barbara Pieretti', Simonetta Gasperoni', Nicoletta Gambini', \\ Gabriele Canucoli', Mario Casini',Tiziana Temellini', Alfredo Vaccaro², Massimo Agostini', \\ Ernesto Delprete'
}

' Dipartimento di Patologia Clinica, Zona Territoriale N 3, Fano (PU)

${ }^{2}$ Dipartimento di Prevenzione, Zona Territoriale $N^{\circ}$ 3, Fano (PU)

Key Words: Toxoplasmosis, Rubella, Citomegalovirus, maternal-fetal infections, seroepidemiologic study

Toxoplasma gondii, Rubella virus and Citomegalovirus infections: seroepidemiology in a pregnant women cohort.

\section{SUMMARY}

Toxoplasma gondii, Rubella virus and Citomegalovirus are responsible for maternal-fetal infections that can cause severe consequences in fetus. The surveillance of these infections in pregnancy is made by specific antibodies detection and when necessary by demostrating the presence of the infectious agent in body fluids.

We have evaluated the seroprevalence of antibodies in a cohort of II00 pregnant women against Toxoplasma gondii, Rubella virus and Citomegalovirus. Our data show a low seroprevalence against Toxoplasma gondii, a suboptimal immunitary coverage against Rubella virus and a significative increase of incidence in Citomegalovirus infection.

The study suggests the need of a multiprofessional and multidisciplinary approach for the right management of these maternal-fetal infections and underlines the pivot role played by Clinical Pathologists.

\section{INTRODUZIONE}

La possibilità di contrarre in gravidanza infezioni trasmissibili capaci di provocare complicanze fetali e/o postnatali anche severe comporta la necessità di realizzare un percorso preventivo, diagnostico e terapeutico multidisciplinare e multiprofessionale previsto anche dal Legislatore nel Decreto Ministeriale del 10 settembre 1998 che individua $\mathrm{i}$ "Protocolli di accesso agli esami di laboratorio e di diagnostica strumentale per le donne in gravidanza e a tutela della maternità" (9). La diagnostica delle infezioni materno-fetali in gravidanza è un'attività particolarmente densa di aspettative per l'utente e comporta un importante impegno di risorse umane e materiali per l'organizzazione sanitaria.

Al Laboratorista spetta il compito di governarla sia dal punto di vista analitico che organizzativo: le indagini di laboratorio svolgono, infatti, un ruolo chiave nel definire lo status immunitario della gravida, nella diagnosi di infezione materna e nello stabilire l'eventuale trasmissione di questa al feto. Il nostro lavoro analizza i risultati sierologici ottenuti in una coorte di gravide afferenti al laboratorio di Patologia Clinica di Fano (Azienda Sanitaria Unica Regionale, Regione Marche,
Zona Territoriale $\mathrm{N}^{\circ}$ 3) nei confronti del Toxoplasma gondii, del virus della Rosolia e del Citomegalovirus essendo questi microrganismi i principali responsabili di infezioni materno-fetali $(5,13,20,26)$.

Lo scopo è stato quello di utilizzare le informazioni sieroepidemiologiche raccolte, dopo averle confrontate con quanto presente in letteratura, principalmente per interventi di medicina preventiva e sanità pubblica.

\section{MATERIALI E METODI}

I dati raccolti si riferiscono ad una coorte di gravide afferenti al nostro laboratorio per la diagnostica sierologica nei confronti di Toxoplasma gondii e Citomegalovirus nel periodo gennaio-maggio 2005 e per tutto l'anno 2005 per il virus della Rosolia.

I campioni presi in considerazione, dopo aver eliminato i test ripetuti, sono stati rispettivamente:

709 per Toxoplasma gondii, 747 per il virus della Rosolia e 419 per il Citomegalovirus, appartenenti a una coorte di gravide di età compresa fra $16 \mathrm{e}$ 44 anni ed età gestazionale fra la quarta e la quarantesima settimana.

La matrice biologica per l'analisi è costituita da 
siero ottenuto centrifugando a $3500 \mathrm{rpm}$ il campione di sangue venoso raccolto in provette Vacuette ${ }^{\circledR}$ Z Serum Sep. Clot Activator Greiner Bio-one da $4 \mathrm{ml}$.

Le indagini sierologiche sono state realizzate determinando gli anticorpi specifici di classe IgG e IgM mediante metodo immunoenzimatico eseguito su strumentazione Mago-Plus (Diamedix $\left.{ }^{\circledR}\right)$.

In particolare $\mathrm{i}$ test per la determinazione delle IgG anti-Toxoplasma, Rosolia e Citomegalovirus impiegano una metodica immunoenzimatica su micropiastra (Enzyme Linked ImmunoSorbent Assay: ELISA).

Lo schema di reazione è simile per i tre test: il siero del paziente è dispensato nella cuvetta di reazione su cui è coattato l'antigene (antigeni inattivati e purificati del virus della Rosolia, antigeni di Toxoplasma e antigeni di Citomegalovirus parzialmente purificato e inattivato con beta-propriolattone).

Dopo una prima incubazione di 30 minuti, un lavaggio elimina il non legato ed è aggiunto un coniugato costituito da anticorpi monoclonali anti-IgG umane legati con perossidasi; segue un'ulteriore incubazione e lavaggio con aggiunta di 3.3',5.5'- tetrametil-benzidina (TMB) substrato della reazione colorimetrica finale che viene bloccata mediante l'aggiunta di una soluzione di acido solforico $0.3 \mathrm{M}$.

I risultati ottenuti in termini di assorbanza a 450 nanometri $(\mathrm{nm})$ sono confrontati con la curva di calibrazione a sei punti prodotta ad ogni seduta analitica ed espressi in Unità Internazionali/ millilitro (U.I./ml).

La tabella 1 riporta i valori di concentrazione delle $\mathrm{IgG}$ specifiche che discriminano i campioni in negativi, indeterminati e positivi.

Tabella I. Valori di concentrazione delle lgG specifiche per Toxoplasma gondii, virus della Rosolia e Citomegalovirus, discriminanti i campioni in negativi, indeterminati e positivi

\begin{tabular}{lccc}
\hline \multicolumn{3}{c}{ Risultato } \\
\hline & Negativo & Indeterminato & Positivo \\
\hline Toxo IgG & $<7 \mathrm{U} . \mathrm{I} . \mathrm{ml}$ & $7-12 \mathrm{U} . \mathrm{I} . \mathrm{ml}$ & $>12 \mathrm{U} . \mathrm{I} . \mathrm{ml}$ \\
\hline Rosolia IgG & $<10 \mathrm{U} . \mathrm{I} . \mathrm{ml}$ & $10-18 \mathrm{U} . \mathrm{I} . \mathrm{ml}$ & $>18 \mathrm{U} . \mathrm{I} . \mathrm{ml}$ \\
\hline CMV IgG & $<9 \mathrm{U} . \mathrm{I} . \mathrm{ml}$ & $9-1 \mathrm{I} \mathrm{U} . \mathrm{I} . \mathrm{ml}$ & $>11 \mathrm{U} . \mathrm{I} . \mathrm{ml}$ \\
\hline
\end{tabular}

La determinazione delle IgM consiste in un metodo immunoenzimatico su micropiastra (ELISA) previa cattura di questa classe di immunoglobuline mediante anticorpi anti-IgM umane fissati al pozzetto di reazione e successiva rivelazione delle IgM specifiche sfruttando la loro capacità di legare l'antigene (antigeni inattivati e purificati del virus della Rosolia, antigeni di Toxoplasma e anti- geni di Citomegalovirus parzialmente purificato e inattivato con beta-propriolattone) coniugato con perossidasi responsabile di una reazione colorimetrica in seguito all'aggiunta del substrato costituito da TMB.

L'assorbanza letta a $450 \mathrm{~nm}$ è confrontata come rapporto con quella prodotta da un controllo interno di cut-off (analizzato in triplicato) e in base a questo rapporto il campione è identificato come positivo, negativo o indeterminato (vedi tabella 2).

Tabella 2. Valori(*) delle IgM specifiche per Toxoplasma gondii, virus della Rosolia e Citomegalovirus, discriminanti i campioni in negativi, indeterminati e positivi

\begin{tabular}{|c|c|c|}
\hline & Risultato & \\
\hline Negativo & Indeterminato & Positivo \\
\hline \multicolumn{3}{|l|}{ Toxo IgM } \\
\hline rapporto $<0.9$ & valore $= \pm 10 \%$ del cut-off & rapporto $>$ I.I \\
\hline \multicolumn{3}{|c|}{ Rosolia IgM } \\
\hline rapporto $<0.9$ & valore $= \pm 10 \%$ del cut-off & rapporto > I.I \\
\hline \multicolumn{3}{|c|}{ CMV IgM } \\
\hline rapporto $<0.9$ & valore $= \pm 10 \%$ del cut-off & rapporto $>$ I.I \\
\hline
\end{tabular}

$\left(^{*}\right)$ i valori derivano dal rapporto tra la densità ottica del siero campione e il valore medio del siero cut-off

I campioni positivi o indeterminati per le $\operatorname{IgM}$ con la metodica ELISA (Diamedix ${ }^{\circledR}$ ) sono stati sottoposti ad analisi con metodo immunoenzimatico a cattura con rivelazione in fluorescenza (ELFA) su MiniVidas ${ }^{\circledR}$ (bioMérieux $\left.{ }^{\circledR}\right)$.

In questa metodica all'immunocattura delle $\operatorname{IgM}$ nel cono di reazione (realizzata tramite anticorpi anti-catena $\mu$ umana) segue l'eventuale legame all'antigene (antigene toxoplasmico di membrana e citoplasmatico, antigene Citomegalovirus AD169, virus della Rosolia inattivato e purificato) a sua volta legato ad un anticorpo monoclonale murino marcato con fosfatasi alcalina che catalizza l'idrolisi del substrato 4-metilumbelliferrilfosfato in 4-metilumbelliferone composto fluorescente la cui intensità di fluorescenza misurata a $450 \mathrm{~nm}$ è proporzionale alla concentrazione degli anticorpi presenti nel campione.

I campioni IgG e IgM positivi sono stati indagati per l'avidità delle IgG.

Il test di avidità delle $\operatorname{IgG}$ si basa sul principio secondo cui le IgG subiscono durante l'evoluzione della risposta immunitaria un aumento dell'affinità di legame per l'antigene che si traduce da un punto di vista diagnostico in un legame antigeneanticorpo meno sensibile all'azione di un agente denaturante quale l'urea.

$\mathrm{Ne}$ deriva che più la produzione di IgG è temporalmente vicina, minore sarà la forza del legame all'antigene e minore sarà l'avidità; ciò permette di datare con buona attendibilità l'infezione.

I test di avidità delle IgG anti-Toxoplasma gondii e anti-Citomegalovirus sono stati eseguiti con 
metodica ELFA su MiniVidas ${ }^{\circledR}$ (bioMérieux ${ }^{\circledR}$ ) nel nostro laboratorio, mentre $\mathrm{i}$ test di avidità delle IgG anti-Rosolia sono stati eseguiti dal Servizio di Virologia del Dipartimento di Malattie Infettive e Microbiologia dell'Azienda Ospedaliera Universitaria-Ospedali Riuniti di Ancona.

La metodica ELFA utilizza una fase solida su cui è fissato l'antigene (antigene toxoplasmico di membrana e citoplasmatico, antigene Citomegalovirus AD169) al quale si fissano le IgG del campione se presenti, segue un lavaggio con e senza urea e rivelazione del legato tramite anticorpi anti-IgG umane coniugati con fosfatasi alcalina che catalizza l'idrolisi del 4-metilumbelliferrilfosfato in 4-metilumbelliferone composto fluorescente la cui intensità di fluorescenza misurata a $450 \mathrm{~nm}$ è proporzionale alla concentrazione degli anticorpi presenti nel campione.

Il rapporto fra i tassi di fluorescenza della reazione con e senza agente denaturante costituisce l'indice di avidità del campione.

In tabella 3 sono riportati i criteri di interpretazione dei test di avidità delle IgG per Toxoplasma gondii, virus della Rosolia e Citomegalovirus.

Tabella 3. Criteri di interpretazione del test di avidità delle lgG per Toxoplasma gondii, virus della Rosolia e Citomegalovirus.

\begin{tabular}{cll}
\hline Toxo & $<$ avidità & $\begin{array}{l}\text { Interpretazione avidità delle IgG } \\
\text { Bassa: infezione primaria entro i } \\
\text { precedenti } 3 \text { mesi }\end{array}$ \\
\cline { 2 - 3 } & $\begin{array}{l}\text { Media: infezione primaria nei prece- } \\
\text { denti } 6 \text { mesi }\end{array}$ \\
\cline { 2 - 3 } & $>25$ & $\begin{array}{l}\text { Alta: infezione non recente, superio- } \\
\text { re ai } 6 \text { mesi precedenti }\end{array}$ \\
\hline Rosolia & $<20$ & $\begin{array}{l}\text { Bassa: infezione primaria entro i pre- } \\
\text { cedenti 2 mesi }\end{array}$ \\
\cline { 2 - 3 } $21-34$ & $\begin{array}{l}\text { Media: infezione primaria nei prece- } \\
\text { denti 4 mesi }\end{array}$ \\
\cline { 2 - 3 } & $>35$ & $\begin{array}{l}\text { Alta: infezione primaria non recente, } \\
\text { superiore ai 4 mesi precedenti }\end{array}$ \\
\hline CMV & $<20$ & $\begin{array}{l}\text { Bassa: infezione primaria recente } \\
\text { contratta entro i precedenti 3 mesi }\end{array}$ \\
\cline { 2 - 3 } $20-80$ & $\begin{array}{l}\text { Media: non consente di chiarire se } \\
\text { 'infezione è entro o oltre i 3 mesi }\end{array}$ \\
\cline { 2 - 3 } & $>80$ & $\begin{array}{l}\text { Alta: infezione primaria non recente } \\
\text { contratta da almeno 3 mesi }\end{array}$ \\
\hline
\end{tabular}

I test di approfondimento diagnostico quali il dosaggio delle IgA specifiche per Toxoplasma gondii (metodica immunoenzimatico a cattura), e la ricerca biomolecolare degli agenti infettivi su sangue, urine, liquido amniotico sono stati condotti dal servizio di Virologia del Dipartimento di Malattie Infettive e Microbiologia dell'Azienda Ospedaliera Universitaria-Ospedali Riuniti di Ancona.

\section{RISULTATI}

Sulla base dei risultati ottenuti possono essere distinti i seguenti quadri immunitari:

- stato recettivo: soggetto $\operatorname{IgG}$ e IgM negativo (sono consigliati controlli mensili dello stato immunitario),

- infezione pregressa: soggetto $\operatorname{IgG}$ positivo e IgM negativo (sono considerate inappropriate le ripetizioni del test),

- stato immunitario non definibile: soggetto con IgG indeterminate e IgM negative (si consiglia ripetizione del test dopo 15 o 30 giorni in base ai risultati precedenti),

- soggetti IgG positivi e IgM positivi: si procede con test alternativi per conferma della positività delle $\mathrm{IgM}$, test di avidità delle $\mathrm{IgG}$, determinazione delle IgA per Toxoplasma gondii e approfondimento diagnostico biomolecolare per definire lo status immunitario

- soggetti IgG negativi e IgM positivi: si procede con test alternativi per la conferma delle IgM, ripetizione del test dopo 15 giorni per valutare la sieroconversione, determinazione delle IgA per Toxoplasma gondii e approfondimento diagnostico biomolecolare per definire lo status immunitario.

In tabella 4 sono riportati (in valore assoluto e percentuale) i risultati ottenuti suddivisi in base allo stato immunitario determinato.

I dati si riferiscono ad una coorte di 1100 gravide di età compresa fra i 16-44 anni, fra la quarta e la quarantesima settimana gestazionale, afferenti al nostro laboratorio per la diagnostica sierologica nei confronti di Toxoplasma gondii e Citomegalovirus (gennaio-maggio 2005) e per il virus della Rosolia (gennaio-dicembre 2005). Sono stati presi in considerazione, dopo aver eliminato i campioni ripetuti, rispettivamente 709 pazienti per Toxoplasma gondii, 747 pazienti per

Tabella 4. Risultati studio sieroprevalenza per Toxoplasma gondii, virus della Rosolia e Citomegalovirus

\begin{tabular}{llllll} 
& Totali & Stato recettivo & $\begin{array}{l}\text { Infezione } \\
\text { pregressa }\end{array}$ & $\begin{array}{l}\text { Stato immunitario } \\
\text { non definibile }\end{array}$ & $\begin{array}{l}\text { Infezione } \\
\text { in atto }\end{array}$ \\
\hline Toxoplasma & 709 & $586(83 \%)$ & $99(14 \%)$ & $23(3 \%)$ & $1(0.2 \%)$ \\
\hline Rosolia & 747 & $81(11 \%)$ & $610(82 \%)$ & $56(7 \%)$ & $/$ \\
\hline Citomegalovirus & 419 & $158(38 \%)$ & $258(61.3 \%)$ & 1 & $3(0.7 \%)$ \\
\hline
\end{tabular}


il virus della Rosolia e 419 pazienti per il Citomegalovirus.

\section{DISCUSSIONE E CONCLUSIONI}

L'analisi dei risultati ottenuti e il loro confronto con quanto presente in letteratura ci permette di fare diverse considerazioni analitiche, organizzative, epidemiologiche e preventive.

Da un punto di vista strettamente analitico va premesso che la confrontabilità con quanto presente in letteratura è affetta da un "bias" non eliminabile dovuto a diverse sensibilità e specificità delle metodiche in commercio conseguenza della scarsa standardizzazione dei diversi test che forniscono valori analitici metodica-dipendenti dovuti all'impiego di antigeni differenti per tipo e preparazione (miscele di antigeni, antigeni ricombinanti, ...) nonché a metodiche diverse (immunoenzimatiche, immunofluorescenza, chemiluminescenza) $(8,21,27)$.

Va inoltre evidenziato come il titolo anticorpale non permette una sicura definizione dello stato immunitario per limiti intrinseci del metodo analitico che per quanto valido sia presenta comunque una zona di valori "borderline": nella nostra realtà questi pazienti (circa il 3\% per Toxoplasma goondii, il $7 \%$ per il virus della Rosolia) sono invitati a ripetere l'esame dopo 15 giorni se si tratta della prima determinazione o 30 giorni per le volte successive.

Questa scelta deriva dal considerare questi pazienti come potenziali esposti al rischio infettivo non potendone garantire uno stato di immunità pregressa.

Per quanto riguarda l'infezione da Toxoplasma gondii da un punto di vista epidemiologico e preventivo esistono diversi indirizzi di politica sanitaria: alcuni Paesi come Francia, Austria, Svizzera e Belgio applicano uno screening prenatale, altri Paesi come Danimarca, Svezia e Brasile attuano uno screening neonatale, mentre in USA, Gran Bretagna e Norvegia lo screening non è raccomandato $(1,3,11)$.

I diversi modelli organizzativi si basano su considerazioni legate al rapporto costo-beneficio dello screening. Tale rapporto, oltre che dai costi diagnostici in senso stretto, dipende dalla prevalenza della infezione (fortemente dipendente dalle condizioni climatiche, igieniche sanitarie e alimentari) ed è influenzato negativamente da alcuni effetti dello screening stesso quali lo stress psicologico per test sierologici positivi, la necessità di approfondimenti diagnostici invasivi, l'aborto di feti sani e la non provata efficacia delle terapie ed i loro effetti collaterali (11).

In Italia la sierologia per la Toxoplasmosi è compresa nel Decreto Ministeriale del 10 settembre
1998 che individua i "Protocolli di accesso agli esami di laboratorio e di diagnostica strumentale per le donne in gravidanza e a tutela della maternità" (9).

Nel mondo la sieroprevalenza della Toxoplasmosi è estremamente variabile essendo come detto fortemente influenzata dalle condizioni climatiche, igieniche sanitarie e alimentari.

I nostri dati indicano una sieroprevalenza nella nostra coorte del 14\%: tale valore è inferiore a quello presente in letteratura per l'Italia (sieroprevelenza soggetti immuni pari al 40-70\%) (10), Francia $(55 \%)$ e Spagna $(28.6 \%)(15,19)$ e simile a quello dei Paesi a più bassa sieroprevalenza quali USA (15\%) (17), UK (10-18\%) $(1,4)$.

Nella nostra realtà l'avere la maggior parte delle gravide esposta al rischio di contrarre la Toxoplasmosi comporta un aumento delle richieste per la sorveglianza dello stato immunitario e impone un'azione multiprofessionale per promuovere, informando e formando, comportamenti igienico-alimentari finalizzati a ridurre al minimo tale rischio.

Studi anche recenti indicano tra le principali fonti di infezione il consumo di carne cruda e semicruda, ortaggi e frutta fresca mentre è stato ridimensionato il ruolo del gatto domestico come portatore della malattia (10).

Ne consegue che si debbano promuovere comportamenti quali: astenersi dalle attività di giardinaggio o lavarsi accuratamente le mani dopo queste, non mangiare carni crude, semicrude e salumi o delegare la pulizia della lettiera possa efficacemente tradursi in infezioni evitate (10).

Sul versante diagnostico e terapeutico se non si conosce il proprio stato immunitario prima della gravidanza entro le prime otto settimane di gestazione, va eseguito il test e ripetuto ogni mese in caso di sieronegatività per evidenziare precocemente l'infezione e attuare un intervento terapeutico tempestivo ed efficace.

Nella nostra casistica abbiamo registrato un singolo caso di sieroconversione che corrisponde ad un tasso dell'1.4\%o leggermente inferiore al 2.4$16 \%$ stimato da Eskild sulla sua popolazione campione (11).

Per quanto riguarda la Rosolia ricordiamo che si tratta di una malattia virale benigna ma quando contratta in gravidanza, soprattutto nel primo trimestre, provoca frequentemente gravi complicanze che vanno dall'aborto spontaneo alla Sindrome da Rosolia Congenita (SRC) (13).

Prima dell'introduzione dei programmi di vaccinazione, la Rosolia era diffusa in tutto il mondo a livelli endemici con epidemie ogni 5-9 anni. Il tasso di SRC durante i periodi endemici era di $0.1-0.2 \%$ nati vivi e durante le epidemie di $1-4 \%$ 
nati vivi. Nei Paesi in cui il vaccino non è stato introdotto la malattia rimane endemica, e nei Paesi in via di sviluppo si stima che ogni anno ci siano circa 100000 casi di SRC $(18,30)$.

Per prevenire l'infezione in gravidanza possono essere adottate differenti strategie vaccinali che prevedono la vaccinazione universale dei bambini nell'infanzia, la vaccinazione delle donne in età fertile e/o delle ragazze adolescenti (vaccinazione selettiva) o una combinazione delle due strategie $(6,30)$.

L'Italia ha introdotto la vaccinazione antirosolia nel 1972, raccomandandola dapprima alle ragazze prepuberi. Dai primi anni '90 la vaccinazione è stata estesa, con l'introduzione del vaccino antimorbillo-parotite-rosolia, a tutti i nuovi nati raccomandando dal 1999 in poi due dosi di vaccino. La vaccinazione delle ragazze prepuberi continua ad essere raccomandata perchè la copertura vaccinale nazionale dei bambini è ancora insoddisfacente e con ampie differenze da regione a regione, pertanto la Rosolia continua a circolare nel nostro Paese con conseguente spostamento verso l'alto dell'età dei casi di malattia. Infatti, negli ultimi anni l'età media dei casi notificati è passata da 9 anni nel periodo 1976-1980 a 12 anni nel periodo 1998-2003 (7).

I casi di SRC registrati in Italia, negli anni 19871991 in cui la notifica della SRC era obbligatoria, sono andati da un minimo di 8 casi nel 1987 ad un massimo di 76 casi nel 1991. Dopo il 1991, non essendo più obbligatoria la notifica, i dati di incidenza della SRC sono quelli relativi alle schede di dimissione ospedaliera con 29 casi nel 1999 e 11 casi nel $2000(12,25)$.

In Italia il "Piano nazionale per l'eliminazione del morbillo e della rosolia congenita" approvato nel 2003 pone tra i suoi obiettivi, in accordo con quanto previsto dall'Ufficio Regionale per l'Europa dell'OMS $(16,23,28,29)$ di ridurre e mantenere entro il 2007 l'incidenza della Rosolia congenita a valori inferiori a 1 caso ogni 100000 nati vivi ed è stato stimato a livello europeo che questo obiettivo è raggiungibile se la proporzione di donne in età fertile suscettibile alla Rosolia è inferiore al $5 \%$.

Un'indagine sieroepidemiologica europea condotta nel 1996 ha evidenziato l'esistenza in Italia di alti livelli di suscettibilità alla Rosolia nella popolazione generale e in particolare una suscettibilità del $10 \%$ nelle ragazze tra 15 e 19 anni e del $7 \%$ nelle donne tra 20 e 39 anni (14).

Purtroppo anche il nostro studio ha evidenziato una percentuale di suscettibilità alla Rosolia dell' $11 \%$.

Nella regione Marche i casi notificati di Rosolia che hanno riguardato le donne in età fertile tra 16 e 45 anni sono stati 43 di 510 casi nel 2000, 164 di 1970 casi nel 2001, 35 di 313 casi nel 2002 e 12 di 315 casi nel 2003; mentre nella Zona Territoriale N. 3 di Fano i casi notificati di Rosolia in donne dai 16 a 45 anni sono stati 4 dei 9 casi nel 2000, 4 dei 46 casi nel 2001 e nessun caso nel periodo 2002-2005 (24).

Anche se negli ultimi anni nella Zona Territoriale N. 3 non sono stati notificati casi di Rosolia nelle donne in età fertile, il dato di un $11 \%$ di donne suscettibili rappresenta comunque un rischio potenziale che deve essere abbattuto attraverso la valutazione della suscettibilità all'infezione (donne in età fertile, prima di un'eventuale gravidanza o in gravidanza) e la vaccinazione (donne prima di un'eventuale gravidanza o nel post-partum).

L'infezione da Citomegalovirus presenta una sieroepidemiologia sovrapponibile a quella presente in letteratura con sieropositività pari al 40-60\% nei Paesi sviluppati per le classi sociali medio alte e dell' $80 \%$ per i ceti meno abbienti (20).

$\mathrm{Da}$ un punto di vista preventivo l'intervento più importante è quello di raccomandare il rispetto delle norme igieniche e se possibile evitare luoghi quali gli asili nido dove il rischio di contrarre il virus è più alto $(2,22)$, visto che al momento non sono disponibili farmaci o vaccini.

La diagnostica sierologica nella sorveglianza in gravidanza dell'infezione da Citomegalovirus svolge un ruolo importante perché permette di individuare e datare l'infezione distinguendo le forme primarie dalle riattivazioni suggerendo gli approfondimenti diagnostici materni, fetali e neonatali e supportando gli interventi terapeutici.

Nella nostra coorte si sono registrate tre sieroconversioni materne per Citomegalovirus: in tutti i casi si è avuta conferma dimostrando la presenza del virus nulle urine materne e in un caso si è evidenziata la presenza del virus su sangue e urine del neonato.

Tre casi accertati di infezione da Citomegalovirus in gravidanza nel periodo di osservazione costituiscono a tutti gli effetti un aumento importante dell'incidenza dell'infezione nella nostra casistica rispetto agli anni precedenti indicando un sensibile aumento nella prima metà del 2005 della circolazione del virus nel nostro territorio.

Tale incidenza non si è riprodotta nei mesi successivi del 2005 dove non abbiamo registrato nuovi casi di infezione da Citomegalovirus nelle gravide esaminate.

Lo studio condotto ci ha permesso di riconsiderare la nostra attività nei confronti di questa particolare tipologia di esami evidenziando come il ruolo del Laboratorista non si esaurisce solo nel comunicare il dato analitico, ma si estende al con- 
trollo dell'appropriatezza della richiesta, alla interpretazione del risultato, alla spiegazione di questo (counselling), alla definizione di un percorso diagnostico organizzato con i centri di riferimento e alla collaborazione con gli altri professionisti coinvolti: igienisti, epidemiologi, ginecologi, pediatri e medici di medicina generale.

A nostro avviso in questo modo si può fornire un servizio in grado di soddisfare le aspettative del singolo utente e promuovere la salute collettiva.

\section{RINGRAZIAMENTI}

Gli autori ringraziano il Dott. Claudio

Piersimoni per la disponibilità e il competente aiuto.

\section{BIBLIOGRAFIA}

1. Ades AE, Parker S, Gilbert R, et al. Maternal prevalence of toxoplasma antibody based on anonymous neonatal serosurvey: a geographical analysis. Epidemiology and Infection, 1993; 110: 127-33.

2. Adler SP. Molecular epidemiology of cytomegalovirus: Viral trasmission among children attendine a day care center, their parents, and caretakers. J Pediatr, 1988, 112: 366-72.

3. Agence Nationale d'Accreditation et d'Evaluation en Santè. La suirvellance biologique femme enceinte en bonne santè et sans antecedent pathologiques. Paris 1996.

4. Allain JP, Palmer CR, Pearson G. Epidemiological study of latent and recent infection by Toxoplasma gondii in pregnant women from a regional population in the UK. Journal of Infection, 1998; 36: 189-96.

5. Britt WJ, Alford CA. Cytomegalovirus. In: Fields Virology, Third Edition. Raven Press, New York 1996; 2494-523.

6. Centers for Disease Control. Rubella prevention: recommendations of the Advisory Committee for Immunization Practices (ACIP). MMWR, 1990; 39 (RR-15): 1-18.

7. Ciofi degli Atti M, Filia A, Revello MG, et al. Rubella control in Italy. Euro Survelliance, 2004; 9: 19-21.

8. Clinical Use and Interpretation of Sierologic Tests for Toxoplasma gondii. Approved Guideline. NCCLS M36, 2004; 24 (6).

9. Decreto Ministeriale, Ministero della Sanità 10 settembre 1998: "Protocolli di accesso agli esami di laboratorio e di diagnostica strumentale per le donne in gravidanza e a tutela della maternità". G.U. n 245, 20 Ottobre 1998.

10. Epicentro. Argomenti di salute: Toxoplasmosi. 2004 (www.epicentro.iss.it).

11. Eskild A, et al. Screening for toxoplasmosis in pregnancy: what is the evidence of reducing a health problem? J Med Screen, 1996; 3: 188.

12. Filia A. Epidemiologia della rosolia in Italia. BEN Notiziario ISS, 2003; 16 (5).

13. Freij BJ, South MA, Sever JL. Maternal rubella and the congenital rubella syndrome. Clin Perinatol, 1988; 15: $247-57$.

14. Gabutti G, Rota MC, Salmaso S, et al. Epidemiology of measles, mumps and rubella in Italy. Epidemiol Infect, 2002; 129: 543-50.

14. Gutierrez-Zufiaurre N, et al. Seroprevalence of antibodies against Treponema pallidum, Toxoplasma gon$d i i$, rubella virus, hepatitis $\mathrm{B}$ and $\mathrm{C}$ virus, and HIV in pregnant women. Enferm Infecc Microbiol Clin, 2004; 22: 512-6.

15. HEALTH 21. The health for all policy framework for the WHO European Region. Copenhagen, WHO Regional Office for Europe, 1999; European Health for All Series No. 6.

16. Jones JL, Kruszon-Moran D, Wilson M. Toxoplasma Gondii infection in United States, 1999-2000. Emergengin Infectious Disease, 2003; 11: 1371-4.

17. Manuale per il Controllo delle Malattie Trasmissibili. Rapporto ufficiale dell'American Public Health Association a cura di David L. Heymann, MD. $18^{\circ}$ Edizione, 2004.

18. Munoz Batet C, Guardia Llobet C, Juncosa Morras T, et al. Toxoplasmosis and pregnancy. Multicenter study of 16362 pregnant women in Barcelona. Med Clin (Barc), 2004 Jun 5; 123 (1): 12-6.

19. Nigro G. L'infezione da Citomegalovirus, EDITEAM, Bologna 2002; 1-43.

20. Ondriska F, Jalili NA, Catar G. Laboratory diagnosis of toxoplasmosis. Bratisl Lek Listy 2000; 101 (5): 294-301.

21. Pass RF, Hutto SC, Ricks R, Cloud GA. Increased rate of cytomegalovirus infection among parents of children attending daycare centers. N Engl J Med 1986; 314: 1414-8.

22. Piano Nazionale per l'eliminazione del morbillo e della rosolia congenita 2003-2007. Ministero della Salute. G.U. n. 297, 23 Dicembre 2003.

23. Piano regionale di attuazione del programma nazionale di eliminazione del morbillo e della rosolia congenita. Regione Marche Delibera ${ }^{\circ}$ 548, 18 Maggio 2004.

24. Piano Nazionale Vaccini (aggiornamento 2005). Ministero della Salute, 10 febbraio 2005.

25. Remington JS, McLeod R, Thulliez P, et al. Toxoplasmosis. In: Infectious Diseases of the Fetus and Newborn Infant. Remington JS, Klein JO, editors. Fifth Edition. The WB Saunders Co, Philadelphia, PA 2001; 205-346.

26. Schinella M, Curci V. La sierologia del complesso Torch. Applicazioni ed interpretazioni. Med Lab, 1998; 6: 157-60.

27. Spika JS, Hanon FX, Wassilak S, et al. Preventing Congenital Rubella Infection in the European Region of WHO: 2001 target. Euro Surveillance, 2004; 9: 4-5.

28. Strategic plan for measles and congenital rubella infection in the European Region of WHO. Copenhagen, WHO Regional Office For Europe, 2003.

29. Weekly Epidemiological Record WHO 19 May 2000; 75: 161-72.

Marco Moretti

Laboratorio Analisi Chimico-Cliniche e Microbiologiche - Zona Territoriale $\mathrm{n}^{\circ} 3$ Fano Viale Vittorio Veneto, 2 - 61032 Fano (PU) Tel. 0721882695 - Fax 0721882304 E-mail: marcomoretti18@libero.it 